\title{
BRIQUETAGEM DE FINOS DE CALCÁRIO
}

\author{
M. R. BARROS ${ }^{1 *}$, A. C. SILVA ${ }^{*}$, E. M. S. SILVA', E. N. MAGALHÃES ${ }^{2}$ e D. Y. MARINHO \\ ${ }^{1}$ Universidade Federal de Goiás, Laboratório de Modelamento e Pesquisa em Processamento Mineral \\ ${ }^{2}$ Instituto Federal de Educação, Ciência e Tecnologia do Triângulo Mineiro - Campus Patos de Minas \\ andre@iceb.ufop.br*
}

Artigo submetido em novembro/2015 e aceito em dezembro/2015

DOI: $10.15628 /$ holos.2015.3714

\section{RESUMO}

Aplicação da tecnologia na agricultura dentro do sistema de produção é realidade principalmente com a abertura de mercados através da globalização. Em diversas áreas da indústria moderna, o calcário é utilizado como corretor da acidez do solo. A calagem é uma prática barata, porém ainda é negligenciada quanto ao seu uso, na adoção da técnica, à definição das doses e às formas de aplicação. A briquetagem consiste na aglomeração de partículas finas através de pressão, auxiliada ou não por aglomerantes, permitindo obtenção de produtos compactados, com forma, tamanho e parâmetros mecânicos adequados. A redução de volume do material, além dos benefícios tecnológicos, permite que materiais finos possam ser transportados e armazenados de forma mais econômica. A recente preocupação ambiental, resultando em leis mais rígidas, além da necessidade de aproveitar economicamente os resíduos e as partículas finas geradas no beneficiamento

de minérios
\end{abstract}

fez com que a briquetagem voltasse a ser uma importante alternativa para aglomerar valor econômico. O objetivo deste trabalho foi aglomerar finos de calcário através da briquetagem ( $92 \%$ abaixo de $500 \#$ ou $25 \mu \mathrm{m}$ ) gerados no processamento do mesmo, variando as dosagens de água (utilizada como agente aglomerante) de $0 ; 5 ; 7,5 ; 10 ; 12,5$ e $15 \%$. O calcário, originário de Lagamar (MG), foi classificado quimicamente como dolomítico tipo $\mathrm{D}$. Os briquetes foram submetidos a testes de queda a 30,60, 90, 120 e $150 \mathrm{~cm}$ de altura. Os melhores resultados encontrados para ensaios de queda foram obtidos com 7,5\% de umidade, com médias de 21 quedas para $30 \mathrm{~cm}$ e 10 quedas para $60 \mathrm{~cm}$ de altura. Tais resultados apresentaram-se favoráveis quando comparados à literatura, a qual cita que para briquetes sem cura, considera-se 3 quedas a 0,3 m como valor razoável.

PALAVRAS-CHAVE: Briquetes, finos, calcário, aglomerante.

\section{LIMESTONE FINES BRICKETTING}

\begin{abstract}
Application of technology in agriculture within the system of production is mostly reality with the opening of markets through globalization. In many areas of modern industry, the limestone, especially in the field, is used as a broker of the acidity of the soil. The liming is a cheap practice, however still is neglected as to its use, in the adoption of the technique, the definition of doses and application forms. The bricketting consists of the agglomeration of fine particles through pressure, aided by agglutination, allowing not only products, but compressed with shape, size and mechanical parameters suitable. The reduction of size of material, in addition to technological benefits, allows thin materials can be transported and stored more economically. The recent environmental concern, resulting in stricter laws, in addition to the need to take advantage of economic
\end{abstract}

waste and fine particles generated in the beneficiation of minerals made briquetting could be an important alternative for agglomerating economic value. The aim of this work was to agglomerate through the limestone fines bricketting ( $92 \%$ below 500 \# or $25 \mu \mathrm{m}$ ) generated in processing the same, varying dosages of water (used as a binder) of $0 ; 5 ; 7.5 ; 10 ; 12.5$ and $15 \%$. The limestone from Lagamar (MG) has been classified chemically as dolomitic type $d$. The brickettes were subjected to tests of fall at 30 , $60,90,120$ and $150 \mathrm{~cm}$ tall. The best results found for drop tests were obtained with $7.5 \%$ humidity, with averages of 21 falls of $30 \mathrm{~cm}$ and 10 falls of $60 \mathrm{~cm}$ height. Such results were favorable when compared to literature, which mentions that for briquettes without cure, considered the falls $0.3 \mathrm{~m} 3$ as reasonable value.

KEYWORDS: Brickettes, slim, limestone, agglomeration. 


\section{INTRODUÇÃO}

Desde tempos remotos, rochas calcárias e dolomitos têm sido amplamente usadas para diversos fins. Desde os tempos das cavernas, como dos tempos bíblicos e da antiguidade pode-se constatar a existência de relatos e comprovações arqueológicos relacionados ao uso dos calcários e dos dolomitos bem como de seus produtos derivados em obras grandiosas e nos empregos domésticos. Para fins voltados a agricultura, não existem determinações permanentes dos teores de carbonato de cálcio a serem aplicados com a finalidade de favorecer a função agricultural, porém quanto maior o teor de $\mathrm{CaCO}_{3}$, menor será a quantidade fundamental para sua utilização (NAHASS e SEVERINO, 2003).

De acordo com Nahass e Severino (2003), a agricultura moderna deve ser voltada ao desenvolvimento sustentável, criando e mantendo a produtividade do solo ao longo prazo. Os sistemas agrícolas, de uma forma geral, empregados aos conceitos de sustentabilidade, isto é, usar sem depredar, de modo a que os recursos naturais, notadamente o solo e a água, possam ser transferidos às gerações futuras, com um legado usufruto, em condições de capacidade produtiva.

Ainda pelos mesmos autores, o uso, com tecnologia apropriada do calcário, protege o ambiente, incrementa a eficiência dos nutrientes e dos fertilizantes, melhora a efetividade de alguns herbicidas e aumenta a produtividade do cultivo. $O$ excesso de acidez é um dos principais obstáculos para a obtenção de altos rendimentos e produtividade dos solos.

A aplicação da tecnologia na agricultura dentro do sistema de produção é uma realidade principalmente com a abertura de novos mercados através da globalização. Dentre as tecnologias disponíveis à agricultura, talvez o que promove maior retorno econômico é o calcário, pois, na região tropical, têm-se, com alta frequência, solos com reação ácida, que limitam o crescimento e produção da maioria das culturas comerciais (PRADO, 2003).

Pesquisas exaustivas e abundantes comprovam que os solos brasileiros são em sua maioria muito ácidos e que a correção de sua acidez pela calagem é imprescindível para se obter uma colheita abundante. Em não sendo utilizada a calagem em alguns solos, os rendimentos de algumas culturas são tão baixos que o seu cultivo se torna economicamente inviável (VOLKWEISS S. et al 1995).

A recente preocupação ambiental, resultando em leis cada vez mais rígidas, além da necessidade de aproveitar economicamente os resíduos e as partículas finas geradas no beneficiamento de minérios fez com que a briquetagem voltasse a ser uma importante alternativa para aglomerar valor econômico (CARVALHO e BRINCK, 2010).

Ainda de acordo com autores Carvalho e Brinck (2010), a briquetagem consiste na aglomeração de partículas finas por meio de pressão, com auxílio ou não de um aglomerante, permitindo a obtenção de um produto não só compactado, porém com forma, tamanho e parâmetros mecânicos adequados. Redução de volumes do material, em alguns casos, além dos benefícios tecnológicos, permite que materiais finos possam ser transportados e armazenados de forma mais econômica.

Portanto, o objetivo deste trabalho foi o aproveitamento do rejeito de mineração do calcário que possui como subproduto um tipo específico conhecido como filler, com granulometria ultrafina que através de processos de aglomeração foram utilizados diminuindo o passivo ambiental, e dessa 
maneira foi dominado o processo de briquetagem do rejeito resultando em briquetes de calcário, com diferentes teores de umidade e aplicados a testes de queda assim que produzidos.

\section{MATERIAL E MÉTODOS}

O experimento foi conduzido no Laboratório de Modelamento e Pesquisa em Processamento Mineral da Universidade Federal de Goiás - Regional Catalão, utilizando calcário "filler" (material tipicamente passante em 500\#), proveniente da região de Lagamar - Minas Gerais.

O primeiro teste realizado foi o estudo da quantidade de água para homogeneização do calcário para conformação do briquete. 0 estudo de umidade dos briquetes foi realizado com 0; 5; 7,$5 ; 10 ; 12,5 ;$ e $15 \%$ de água em peso. Na porcentagem de $12,5 \%$ a conformação do briquete apresentou-se pouco mais difícil, pois apenas a água extravasa para fora do cilindro quando aplicada a pressão desejada, porém a retirada no briquete da fôrma ainda era possível e a com a porcentagem de $15 \%$ de umidade não foi possível à fabricação do briquete, pois o material depois que aplicada à pressão necessária, transbordava totalmente para fora do cilindro.

O calcário utilizado nos experimentos teve sua massa específica determinada através de picnômetria, sendo a análise granulométrica do mesmo realizada por peneiramento a úmido, efetuado em triplicata. Massas de $50 \mathrm{~g}$ de calcário foram peneiradas com adição de solução de metassilicato de sódio a $1 \%$. Uma amostra do calcário foi enviada para análise química, realizada pelo Instituto de Ciências Agrárias da Universidade Federal de Uberlândia.

Avaliou-se o potencial de aglomeração do calcário por meio da briquetagem, com água como agente aglomerante, utilizando uma massa de calcário de $15 \mathrm{~g}$ em cada composição elaborada. Após homogeneização da mistura de calcário e água a massa resultante foi colocada em uma briquetadeira (Figura 1) e aplicada compressão uniaxial na mesma, de modo a promover a aglomeração do material.
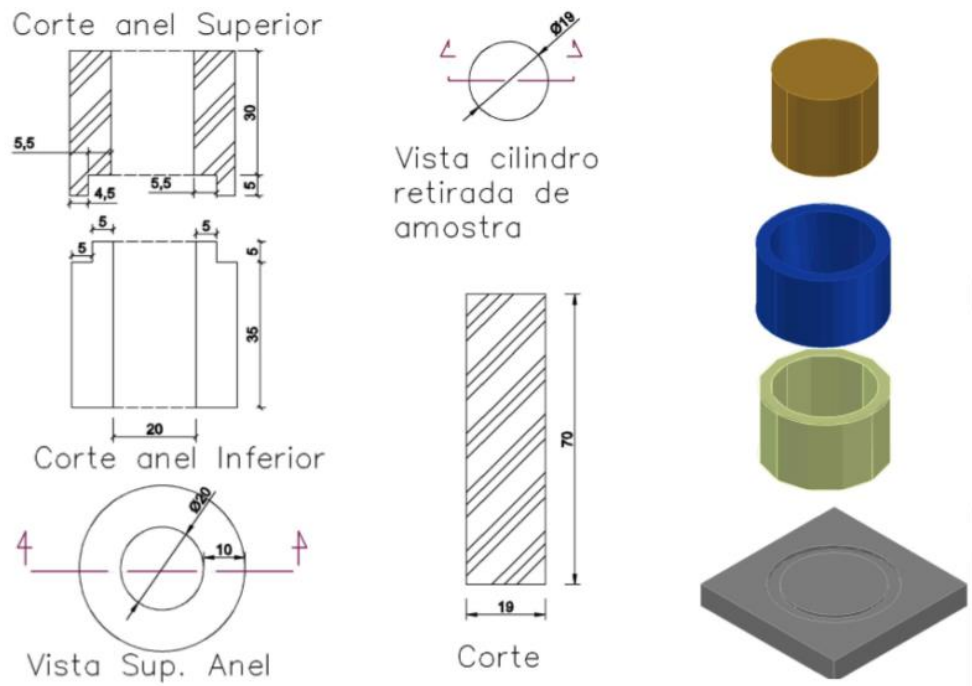

Figura 1: Cortes e vistas do cilindro utilizado para produção dos briquetes.

Para a pesagem do material, calcário, foi utilizada uma balança SHIMADZU. A adição da água foi realizada com pipetas graduadas para garantia do correto volume conforme a quantidade de líquido necessária. Em um béquer de $250 \mathrm{~mL}$ foi adicionado as $15 \mathrm{~g}$ de calcário e a quantidade de 
água parcelada em 3 vezes para garantir que a homogeneização da mistura fosse a melhor possível, sendo esta etapa realizada com ajuda de uma espátula.

Após a determinação da quantidade de água para conformação dos briquetes os mesmos foram produzidos para realização dos testes de queda. Os testes foram realizados através da queda livre dos briquetes em alturas padronizadas de 30,60, 90, 120 e $150 \mathrm{~cm}$ através da colocação de um suporte metálico graduado que garantia o padrão de queda. $O$ briquete foi submetido à altura de $30 \mathrm{~cm}$ e aferido seu peso na balança de precisão acima supracitado, sendo o mesmo briquete após pesado recolhido e submetido à altura posterior repetindo este processo até que o mesmo perdesse $5 \%$ de sua massa original. Por fim, os briquetes foram pesados e medidos (diâmetro e altura), de modo a calcular a massa específica de cada um deles.

\section{RESULTADOS E DISCUSSÃO}

A massa específica encontrada nos ensaios de picnômetria foi de $2,765 \mathrm{~g} / \mathrm{cm}^{3}$, resultado obtido que pode ser confirmado por valores encontrados na literatura $\left(2,72\right.$ a $\left.2,87 \mathrm{~g} / \mathrm{cm}^{3}\right)$ (SAMPAIO e ALMEIDA, 2008). A análise granulométrica de calcário é apresentada na Figura 2.

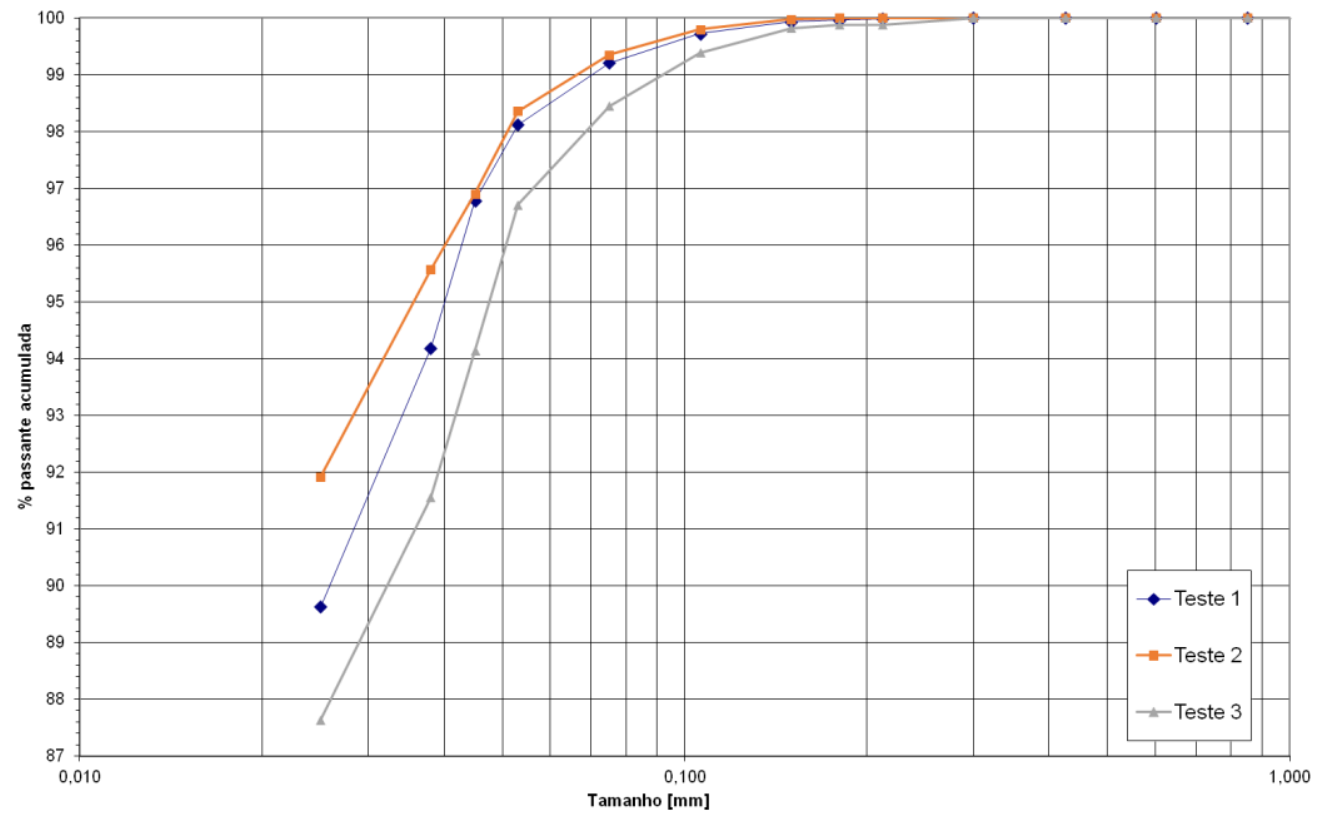

Figura 2: Análise granulométrica das amostras de calcário.

A partir da análise do gráfico acima pode-se concluir que, pela média dos três testes, que uma quantidade de material superior a $90 \%$ possui granulometria passante na peneira de $500 \#$, evidenciando-se assim as dimensões extremamente pequenas das partículas de calcário utilizado nos ensaios de aglomeração.

A Tabela 1 apresenta o resultado da análise química da amostra de calcário utilizado nos testes, sendo o mesmo classificado como calcário dolomítico D. A classificação foi obtida baseandose no teor de óxido de magnésio (Calcítico: < $5 \% \mathrm{MgO}$, Dolomítico: > $=5 \% \mathrm{MgO}$ ) e o grupo quanto ao PRNT (Poder Relativo de Neutralização Total) - grupo D (>90\%).

Tabela 2: Análise química da amostra de calcário. 


\begin{tabular}{cccccccc}
\hline $\mathrm{CaO}$ & $\mathrm{CaCO}_{3}$ & $\mathrm{MgO}$ & $\mathrm{MgCO}_{3}$ & $\mathrm{PN}$ & $\mathrm{ER}$ & $\mathrm{PRNT}$ & $\begin{array}{c}\text { Sílica e } \\
\text { insolúveis }\end{array}$ \\
\hline 33,6 & 59,8 & 11,1 & 23,3 & 100,0 & 99,6 & 99,6 & Ns \\
\hline
\end{tabular}

A Figura 3 apresenta vistas dos briquetes de calcário a partir do calcário filler com forma, tamanho e parâmetros mecânicos adequados adquiridos após o processo de briquetagem antes submissão aos testes de queda. E as figuras $\mathrm{c}$ e $\mathrm{d}$, mostram briquetes submetidos a testes de quedas a $30 \mathrm{~cm}$ de altura, até perderem $5 \%$ da massa. As Figuras apresentam briquetes com 7,5\% de umidade, os quais demonstraram maiores resistências mecânicas.

De acordo com a análise da Figura 4, pode-se perceber que há uma redução no número de quedas quando se eleva as alturas nos testes, como esperado. Sem a adição de aglomerantes (Figura 4a) o briquete se mostrou frágil, suportando menos que 5 quedas a $30 \mathrm{~cm}$ e reduzindo sua resistência mecânica nas demais. Já com a adição de água como aglomerante (Figura 4b), os briquetes obtiveram maior resistência mecânica nas primeiras duas alturas, demonstrando assim seu potencial na briquetagem deste material.

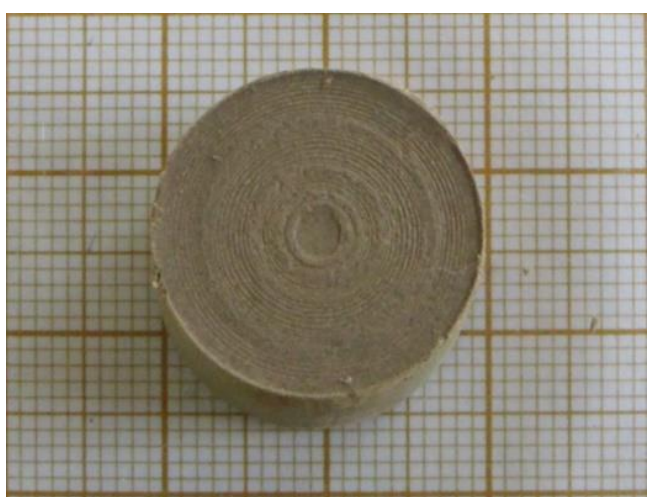

(a)

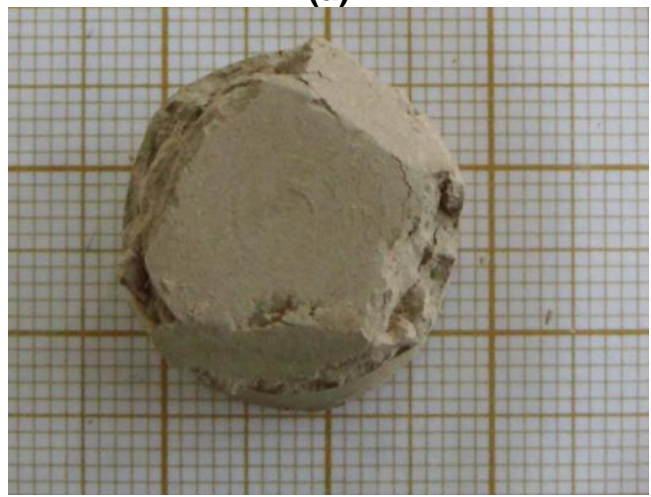

(c)

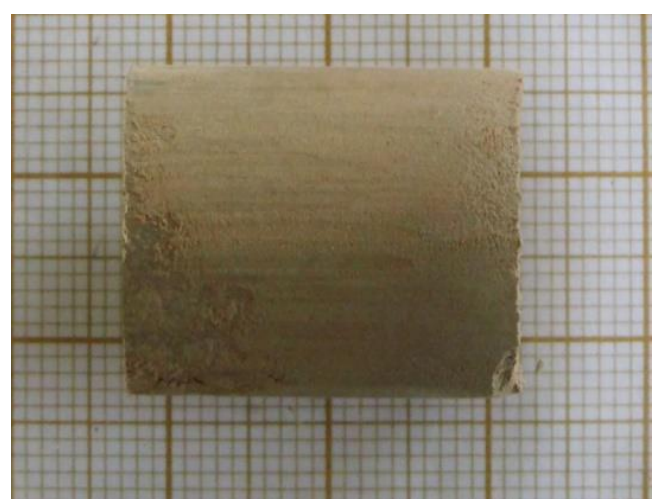

(b)

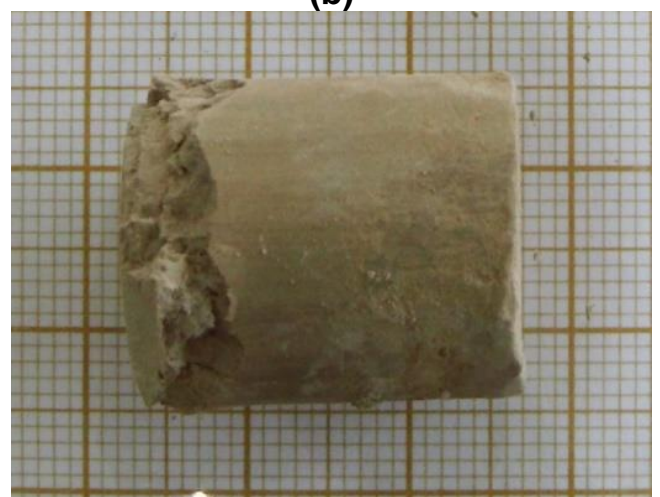

(d)

Figura 3: Vistas dos briquetes: (a) superior e (b) lateral antes do teste de queda, e (c) superior e (d) lateral após o teste de queda. 


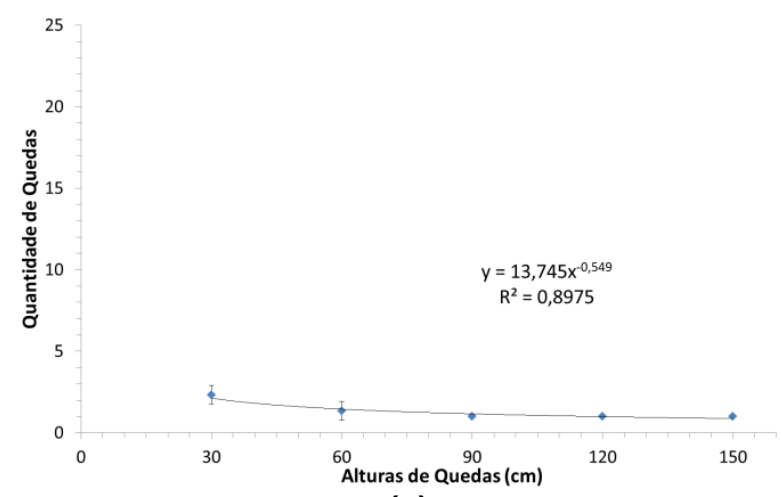

(a)

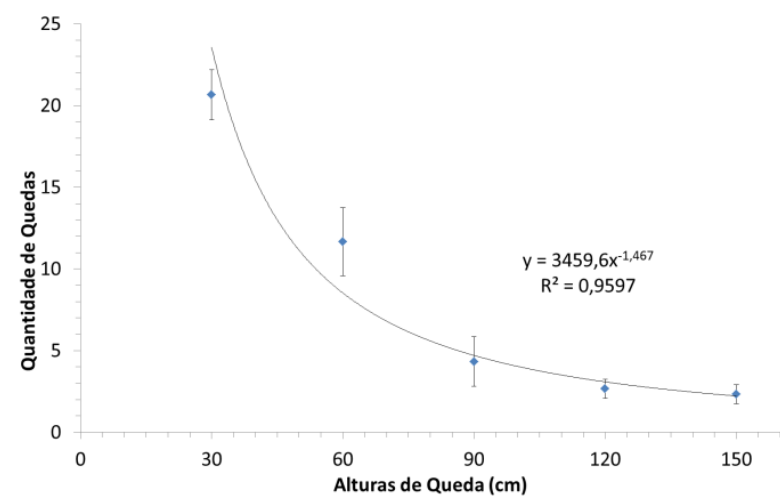

(c)

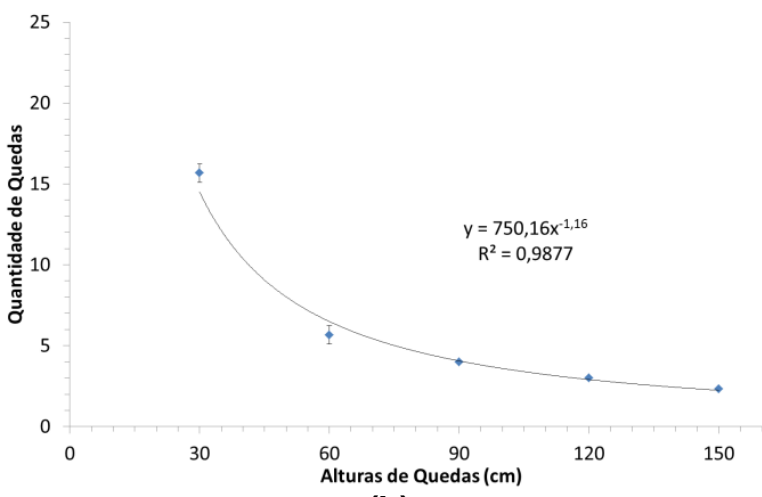

(b)

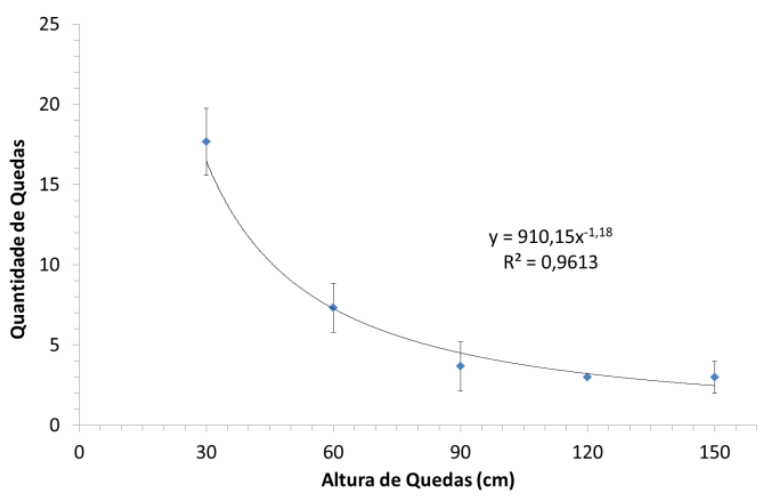

(d)

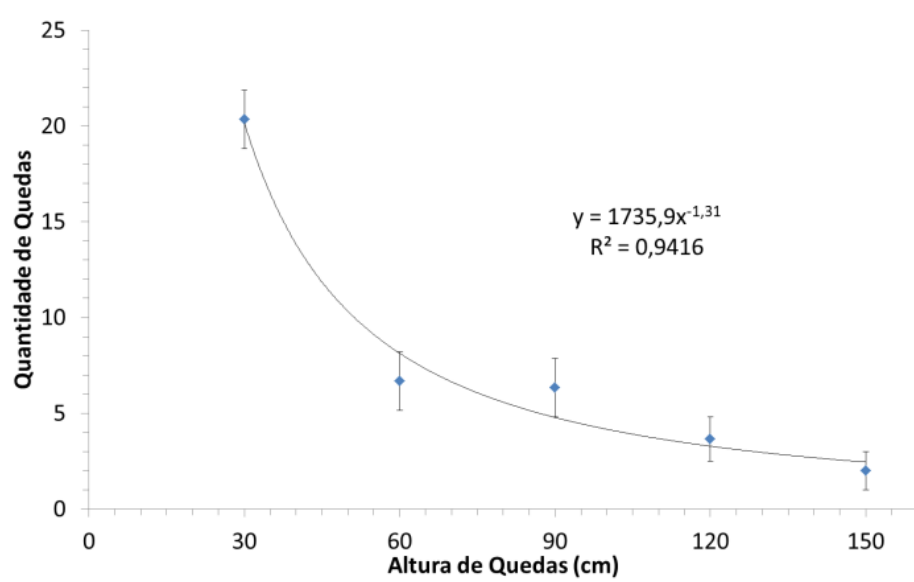

(e)

Figura 4: Quantidade de quedas em relação às alturas de queda: (a) 0; (b) 5; (c) 7,5; (d) 10; (e) 12,5\% de umidade.

$\mathrm{Na}$ Figura 5, a massa específica comparada a umidade, mostra que quanto maior a porcentagem de água presente no briquete maior será sua densidade. A massa específica dos briquetes não chegou ao resultado da picnometria de $2,765 \mathrm{~g} / \mathrm{cm}^{3}$, pois se entende que no material, durante a aplicação de pressão, os poros foram preenchidos por água, que possui massa especifica de $1 \mathrm{~g} / \mathrm{cm}^{3}$, dessa forma os briquetes não teriam a mesma massa específica definida pelo teste do picnômetro, justificando sua densidade menor que o esperado. 


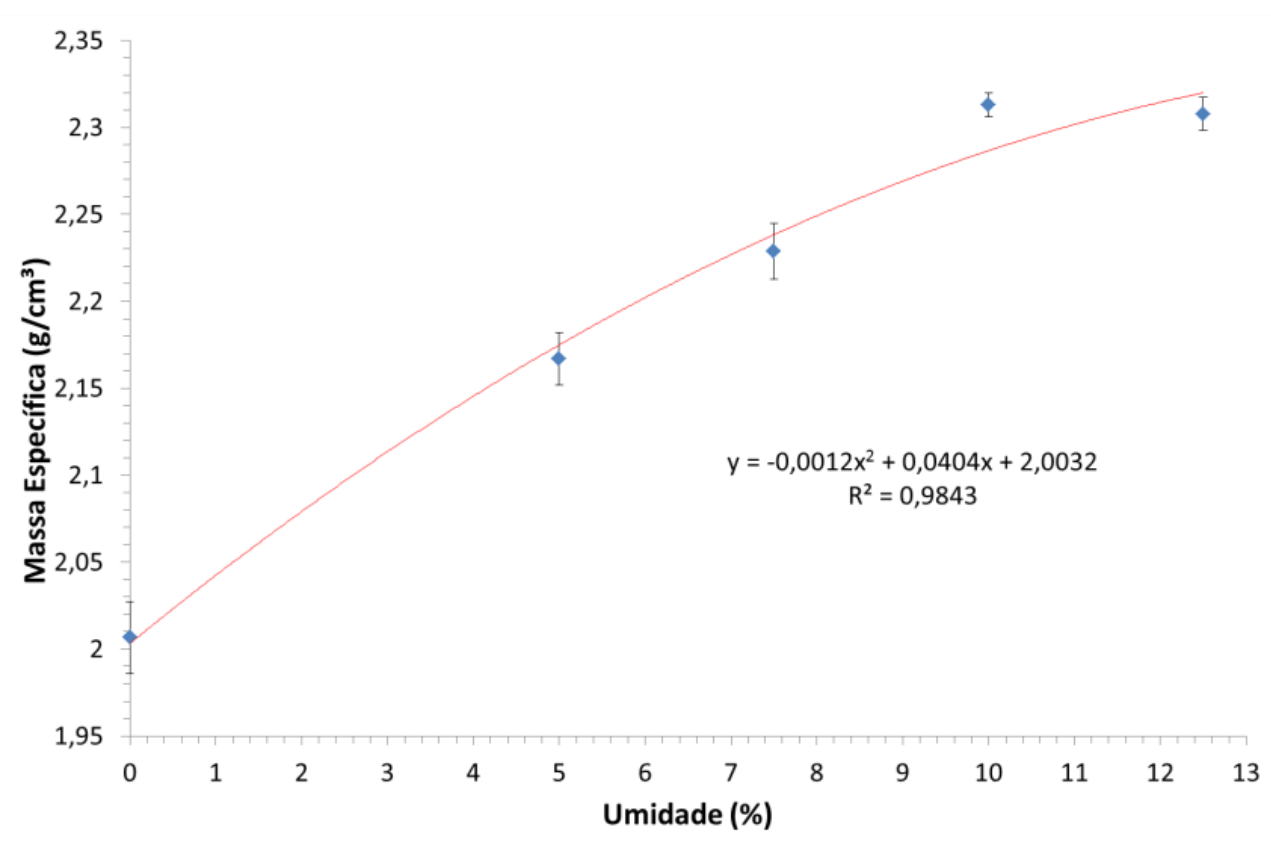

Figura 5: Variação da massa específica dos briquetes em relação à umidade.

\section{CONCLUSÕES}

Diante dos resultados pode-se comprovar que a briquetagem é possível utilizando água como aglomerante. Os melhores resultados encontrados para avaliar resistência mecânica foram registrados com os teores de umidade de $7,5 \%$ e $12,5 \%$.

Nesse caso os briquetes apresentaram o melhor desempenho nas alturas de 30 e $60 \mathrm{~cm}$, a $7,5 \%$ de umidade com médias de 21 e 9 quedas respectivamente, e 21 e 6 quedas também a 30 e $60 \mathrm{~cm}$ de altura na devida ordem a $12,5 \%$ de água. Os testes foram efetuados até que os briquetes perdessem $5 \%$ de sua massa.

Considera-se uma perda de massa de até $5 \%$ como um valor ótimo para estes tipos de briquetes, sendo considerados ruins quando as perdas são superiores a $10 \%$. Tais resultados apresentaram-se favoráveis quando comparados à literatura, a qual cita que para briquetes sem cura, considera-se 3 quedas a 0,3 m como um valor razoável (CARVALHO E BRINCK, 2010). O resultado obtido é sete vezes maior que o mínimo necessário. Ainda além, quanto maior a umidade presente no briquete (até $12,5 \%$ em peso), maior é a resistência nas primeiras duas alturas no teste de queda (30 e $60 \mathrm{~cm}$ ).

\section{AGRADECIMENTOS}

Agradecemos às agências de fomento CNPq, CAPES e FAPEG pelo auxílio financeiro, primordial para a realização do presente estudo, bem como à Cala Calcário Lagamar Ind. Comércio por ceder o calcário utilizado no estudo e a Universidade Federal de Goiás.

\section{REFERÊNCIAS BIBLIOGRÁFICAS}

1. CARVALHO, E. A.; BRINCK, V. Briquetagem. Centro de Tecnologia Mineral Ministério da Ciência 
e Tecnologia. Coordenação de Processos Minerais - COPM, Rio de Janeiro, 2010.

2. NAHASS, S.; SEVERINO, J. Calcário Agrícola no Brasil. CETEM/MCT. Rio de Janeiro, 2003.

3. PRADO, R. M. A calagem e as propriedades físicas de solos tropicais: revisão de literatura. Departamento de Solos e Adubos, Faculdade de Ciências Agrárias e Veterinárias da Universidade Estadual Paulista. Revista Biociências, Taubaté, v.9, n.3, p.7-16, jul.-set. 2003.

4. SAMPAIO, J. A.; ALMEIDA, S. L. M. Rochas e Minerais Industriais. In: LUZ, A. B.; LINS, F. A. F. $2^{\circ}$ edição. Capítulo 15, Calcário e Dolomito Rio de Janeiro: CETEM/MCT, 2005.

5. VOLKWEISS, S. J.; TEDESCO, M. J.; GIANELLO, C.; BISSANI, C. A.; BOHNEN, H. Análise de solo, plantas e outros materiais. 2. ed. Porto Alegre: UFRGS, 1995. 174 p. (Boletim Técnico, 5). 